\title{
Learning Neighborhood Discriminative Manifolds for Video-Based Face Recognition
}

\author{
John See ${ }^{1}$ and Mohammad Faizal Ahmad Fauzi ${ }^{2}$ \\ 1 Faculty of Information Technology, Multimedia University, \\ Persiaran Multimedia, 63100 Cyberjaya, Selangor, Malaysia \\ 2 Faculty of Engineering, Multimedia University, \\ Persiaran Multimedia, 63100 Cyberjaya, Selangor, Malaysia \\ \{johnsee, faizal1\}@mmu. edu.my
}

\begin{abstract}
In this paper, we propose a new supervised Neighborhood Discriminative Manifold Projection (NDMP) method for feature extraction in video-based face recognition. The abundance of data in videos often result in highly nonlinear appearance manifolds. In order to extract good discriminative features, an optimal low-dimensional projection is learned from selected face exemplars by solving a constrained least-squares objective function based on both local neighborhood geometry and global manifold structure. The discriminative ability is enhanced through the use of intra-class and inter-class neighborhood information. Experimental results on standard video databases and comparisons with state-of-art methods demonstrate the capability of NDMP in achieving high recognition accuracy.
\end{abstract}

Keywords: Manifold learning, feature extraction, video-based face recognition.

\section{Introduction}

Recently, manifold learning has become an increasingly growing area of research in computer vision and pattern recognition. With the rapid development in imaging technology today, it plays an important role in many applications such as face recognition in video, human activity analysis and multimodal biometrics, where the abundance of data often demands better representation.

Typically, an image can be represented as a point in a high-dimensional image space. However, it is common presumption that the perceptually meaningful structure of the data lies on or near a low-dimensional manifold space [1]. The mapping between high- and low-dimensional spaces is accomplished through dimensionality reduction. This remains a challenging problem for face data in video, where large complex variations between face images can be better represented by extracting good features in the low-dimensional space.

In this paper, we propose a novel supervised manifold learning method called Neighborhood Discriminative Manifold Projection (NDMP) for feature extraction in video-based face recognition. NDMP builds a discriminative eigenspace 
projection of the face manifold based on the intrinsic geometry of both intraclass and inter-class neighborhoods. For each training video, a set of face representative exemplars are automatically selected through clustering. With these exemplars, an optimal low-dimensional projection is learned by solving a constrained least-squares (quadratic) objective function using local neighborhood and global structural constraints. A compact generalized eigenvalue problem is formulated, where new face data can be linearly projected to the feature space. Finally, the test video sequences are classified using a probabilistic classifier.

\section{Related Work}

Classical linear dimensionality reduction methods such as Principal Component Analysis (PCA) [2, Multidimensional scaling (MDS) [3] and Linear Discriminant Analysis (LDA) 4] are the most popular techniques used in many applications. These linear methods are clearly effective in learning data in simple Euclidean structure. PCA learns a projection that maximizes its variance while MDS preserves pairwise distances between data points in the new projected space. With additional class information, LDA learns a linear projection that maximizes the ratio of the between-class scatter to the within-class scatter. The biggest drawback of these methods is that they fail to discover the intrinsic dimension of the image space due to its assumption of linear manifolds. Also, overfitting remains a common problem in these methods.

The emergence of nonlinear dimensionality reduction methods such as Locally Linear Embedding (LLE) [5] and Isomap [6] signalled the beginning of a new paradigm of manifold learning. These methods are able to discover the underlying high-dimensional nonlinear structure of the manifold in a lower dimensional space. LLE seeks to learn the global structure of a nonlinear manifold through a linear reconstruction that preserves its local neighborhood structure. Isomap is similar in nature, but geodesic distances between points are computed before reducing the dimensionality of space with MDS.

The main disadvantage of these methods is that they cannot deal with the out-of-sample problem - where new data points cannot be projected onto the embedded space. This limits their potential usage for classification and recognition tasks. Several works [78] reported good recognition rates in a video-based face recognition setting by using LLE to build a view-based low-dimensional embedding for exemplar selection, but stops short of utilizing it for feature representation.

In recent developments, various manifold learning methods such as Locality Preserving Projections (LPP) [9], Marginal Fisher Analysis (MFA) [10] and Neighborhood Preserving Embedding (NPE) 11 have been proposed to solve the out-of-sample problem. These methods resolve this limitation by deriving optimal linear approximations to the embedding using neighborhood information in the form of neighborhood adjacency graphs (with simple binary or heat kernel functions). While these methods can be performed in supervised mode with additional class information, their discriminative ability is only limited to 
neighborhood point relationships by graph embedding. It does not distinguished between neighborhood structures created by intra-class and inter-class groups of points within the same manifold, which can be very disparate in nature.

The major contribution of this paper centers upon the novel formulation of a neighborhood discriminative manifold learning method that exploits the intrinsic local geometry of both intra-class and inter-class neighborhoods while preserving its global manifold structure.

\section{NDMP Algorithm}

In this section, we give some motivations behind our work, followed by an overview of the Locally Linear Embedding (LLE) algorithm and a detailed description of the proposed NDMP method. Brief remarks on some closely-related techniques are also provided.

\subsection{Motivations}

The rich literature of manifold learning methods offers many potential avenues for improving existing shortcomings. With this, we want to highlight two main motivations behind our work.

1. While recent works 91011 have addressed the out-of-sample problem with added discriminative properties, they do not discriminate between withinclass and between-class neighborhood structures while keeping the global manifold fitted. Although similar to the NPE 11 in terms of its theoretical foundation (based on LLE [5]), our final objective function is different.

2. Most works reported impressive results on data sets with limited face variations such as ORL, Yale and UMIST datasets [12. In video sequences, the large amount of face variations may prove to be a challenging task due to the difficulty in finding good meaningful features.

\subsection{Locally Linear Embedding (LLE)}

Assume $X=\left\{x_{i} \in \Re^{D} \mid i=1, \ldots, N\right\}$ represent the input data in Euclidean space consisting of $N$ face samples in $D$ dimensions, belonging to one of $C$ classes $\{c \mid c \in\{1, \ldots, C\}\}$. The LLE algorithm can be summarized into 3 main steps:

Step 1: For each point $x_{i}$, compute its $k$ nearest neighbors.

Step 2: Compute the reconstruction weights $W$ that best reconstruct each point $x_{i}$ from its neighbors by minimizing the cost function

$$
\epsilon_{r e c}(W)=\sum_{i=1}^{N}\left\|x_{i}-\sum_{j=1}^{k} W_{i j} x_{j}\right\|^{2},
$$


where $W_{i j}$ is the reconstruction weight vector, subject to constraints $\sum_{i=1}^{N} W_{i j}=$ 1 and $W_{i j}=0$ if $x_{i}$ and $x_{j}$ are not neighbors.

Step 3: Compute the embedding coordinates $y_{i}$ best reconstructed by the optimal reconstruction weight $W$ by minimizing the cost function

$$
\epsilon_{e m b}(y)=\sum_{i=1}^{N}\left\|y_{i}-\sum_{j=1}^{k} W_{i j} y_{j}\right\|^{2},
$$

subject to constraints $\sum_{i=1}^{N} y_{i}=1$ and $\sum_{i=1}^{N} y_{i} y_{i}^{T} / N=I$ where $I$ is an identity matrix. The new coordinates in embedded space, $Y=\left\{y_{i} \in \Re^{d} \mid i=1, \ldots, N\right\}$ is a $d \times N$ matrix, where the dimensionality of the new embedded points, $d<D$.

\subsection{Neighborhood Discriminative Manifold Projection (NDMP)}

Considering the large amount of face variations in each training video sequence, we first apply LLE to reduce the dimensionality of the data. Then, faces in each training video sequence are grouped into clusters using hierarchical agglomerative clustering (HAC) 13. For each cluster, the face that is nearest to the cluster mean is selected as an exemplar, or a representative image of the cluster. Similar to these approaches [78, the subject in each training video is represented by a set of $M$ exemplars, which are automatically extracted from the video. Features will be extracted from the exemplar sets using the NDMP method. Finally, subjects in the test video sequences are identified using a probabilistic classifier.

In the proposed NDMP method, we first construct two sets of reconstruction weights - one each for the intra-class and inter-class neighborhoods, unlike the NPE which uses only one set of weights. The optimal projection is then formulated as a constrained minimization problem.

Construction of Intra-class and Inter-class Neighborhood Subsets. For clarity, the exemplars are also regarded as data points in most part of the paper. Let all data points in a local neighborhood $\Psi$ comprise of two disjointed neighborhood subsets - intra-class subset $\left\{\psi_{p} \mid p=1, \ldots, k\right\}$ and inter-class subset $\left\{\psi_{q}^{\prime} \mid q=1, \ldots, k^{\prime}\right\}$. Compute the $k$ nearest intra-class neighbors and $k^{\prime}$ nearest inter-class neighbors of each point $x_{i}$. A point $x_{j}$ is an intra-class neighbor of point $x_{i}$ if they belong to the same class $\left(c_{i}=c_{j}\right)$. Similarly, point $x_{j}$ is an inter-class neighbor of point $x_{i}$ if they do not belong to the same class $\left(c_{i} \neq c_{j}\right)$.

It is possible for a local neighborhood to have unequal number of intra-class and inter-class nearest neighbors. However, for the sake of uniformity of weight matrices, we fix $k=k^{\prime}$. Due to class set limitation, the number of intra-class and inter-class neighbors is restricted to a maximum of $M-1$, where $M$ is the number of exemplars in a class. 
Formulation of Neighborhood Reconstruction Weights. Based on Eq. (1), we formulate two reconstruction cost functions to obtain neighborhood reconstrution weights that best reconstruct each point $x_{i}$ based on its type of neighbors. Both intra-class reconstruction weight matrix $W^{r}$ and inter-class reconstruction weight matrix $W^{e}$ can be computed by minimizing the respective cost functions:

$$
\begin{aligned}
& \epsilon_{r e c}\left(W^{r}\right)=\sum_{i=1}^{N}\left\|x_{i}-\sum_{j=1}^{k} W_{i j}^{r} x_{j}\right\|^{2}, \\
& \epsilon_{r e c}\left(W^{e}\right)=\sum_{i=1}^{N}\left\|x_{i}-\sum_{j=1}^{k^{\prime}} W_{i j}^{e} x_{j}\right\|^{2} .
\end{aligned}
$$

Both Eqs. (3) and (4) can be computed in closed form by determining the optimal weights through the local covariance matrix [5]. Alternatively, it can also be solved using a linear system of equations.

Formulation of Optimal Projection. In this step, an optimal projection is learned to enable new data points to be linearly embedded in the NDMP feature space. Typically, a linear subspace projection

$$
Y=A^{T} X
$$

maps the original data points $X$ to the projected coordinates in embedded space $Y$ by a linear transformation matrix $A=\left\{a_{i} \in \Re^{D} \mid i=1, \ldots, d\right\}$.

Similar to NPE algorithm [11, the intra-class cost function can be formulated by expanding the least-squares term:

$$
\begin{aligned}
\epsilon_{e m b}^{r}(Y) & =\sum_{i=1}^{N}\left\|y_{i}-\sum_{j=1}^{k} W_{i j}^{r} y_{j}\right\|^{2} \\
& =\left\|Y-\sum_{i=1}^{N} \sum_{j=1}^{k} W_{i j}^{r} y_{j}\right\|^{2} \\
& =\operatorname{Tr}\left(Y\left[\delta_{i j}-2 W^{r}+\left\|W^{r}\right\|^{2}\right] Y^{T}\right) \\
& =\operatorname{Tr}\left(Y\left(I-W^{r}\right)^{T}\left(I-W^{r}\right) Y^{T}\right) \\
& =\operatorname{Tr}\left(Y M^{r} Y^{T}\right),
\end{aligned}
$$

where $\operatorname{Tr}\{$.$\} refers to the trace of the matrix and the orthogonal intra-class$ weight matrix

$$
M^{r}=\left(I-W^{r}\right)^{T}\left(I-W^{r}\right) .
$$

Likewise, the inter-class cost function and its orthogonal inter-class weight matrix is derived as

$$
\begin{aligned}
\epsilon_{\text {emb }}^{e}(Y) & =\operatorname{Tr}\left(Y M^{e} Y^{T}\right), \\
\text { where } M^{e} & =\left(I-W^{e}\right)^{T}\left(I-W^{e}\right) .
\end{aligned}
$$


Substituting Eq. (5) to both cost functions yields:

$$
\begin{aligned}
& \epsilon_{e m b}^{r}(X)=\operatorname{Tr}\left(A^{T} X M^{r} X^{T} A\right) \\
& \epsilon_{e m b}^{e}(X)=\operatorname{Tr}\left(A^{T} X M^{e} X^{T} A\right) .
\end{aligned}
$$

Motivated by Fisher's discrimination criterion [4, the objective function can be formulated to incorporate discriminative property that enables the compaction of intra-class neighborhood and the dispersion of inter-class neighborhood (see Fig. 1 for illustration). The intra-class cost function, $\epsilon_{e m b}^{r}$ can be minimized so that the overall weighted pairwise distances between intra-class neighbors in embedded space are reduced.

Since the total sum of weights is subjected to $\sum_{i=1}^{N} W_{i j}=1$ or $\operatorname{Tr}(W)=I$, the inter-class cost function can be formulated as a local constraint,

$$
A^{T} X M^{e} X^{T} A=I \text {. }
$$

In order to maintain global rotational invariance within the embedding structure, another constraint is used for further optimization. From Step 3 of the LLE algorithm, rotational invariance is achieved by subjecting $\sum_{i=1}^{N} y_{i} y_{i}^{T} / N=I$ or $Y Y^{T} / N=I$, resulting in a global constraint,

$$
A^{T} X X^{T} A=N I \text {. }
$$

Modeling a constrained optimization problem involving two constraints results in the following Lagrangians:

$$
\begin{aligned}
L_{\ell}\left(\lambda_{\ell}, A\right) & =A^{T} X M^{r} X^{T} A+\lambda_{\ell}\left(I-A^{T} X M^{e} X^{T} A\right) \\
L_{g}\left(\lambda_{g}, A\right) & =A^{T} X M^{r} X^{T} A+\lambda_{g}\left(N I-A^{T} X X^{T} A\right) .
\end{aligned}
$$

By forming two equations from their derivatives w.r.t. $A$ and solving them simultaneously, it can be easily shown that $\lambda_{\ell}=\lambda_{g}=\lambda$ based on the constraints in Eqs. (12) and (13). Thus, the corresponding unified Lagrangian,

$$
L^{\prime}(\lambda, A)=A^{T} S A+\lambda\left((N+1) I-A^{T} C_{\ell} A-A^{T} C_{g} A\right),
$$

where $S=X M^{r} X^{T}, C_{\ell}=X M^{e} X^{T}$ and $C_{g}=X X^{T}$.

Setting its gradient to zero,

$$
\frac{\partial L^{\prime}}{\partial A}=0 \Rightarrow 2 S A-\lambda\left[2 C_{\ell} A+2 C_{g} A\right]
$$

we can then rewrite it as a generalized eigenvalue problem,

$$
S A=\lambda\left[C_{\ell}+C_{g}\right] A \text {. }
$$

Note that matrices $S, C_{\ell}$ and $C_{g}$ are all symmetric and semi-positive definite. The optimal embedding $A$ is solved by taking $d$ eigenvectors associated with the $d$ smallest eigenvalues $\left(\lambda_{1}<\ldots<\lambda_{d}\right)$, where $d<D$.

In a generalized case, a constraint tuning parameter $\beta=\{\beta \mid 0 \leq \beta \leq 1\}$ can be introduced to allow both local and global constraints to be adjusted according to importance,

$$
S A=\lambda\left[\beta C_{\ell}+(1-\beta) C_{g}\right] A .
$$

For equal contribution from both constraints, we can fix $\beta=0.5$. 

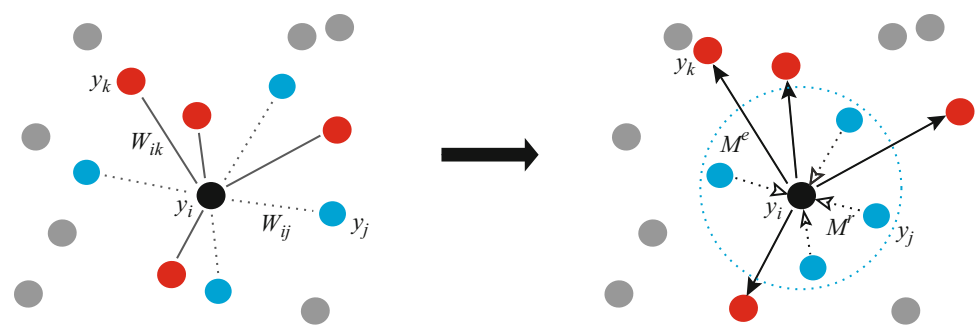

Fig. 1. A data point (black) is embedded in NDMP-space using weights from intraclass (blue) and inter-class (red) neighborhoods. Solving the constrained minimization problem leads to distinction between intra-class and inter-class structures.

\section{Experimental Results}

The proposed NDMP method was tested on two standard video face datasets: Honda/UCSD [14] and CMU MoBo [15] in order to ensure a comprehensive evaluation was conducted. The first dataset, Honda/UCSD, which was collected for video-based face recognition, consists of 59 video sequences of 20 different people (each person has at least 2 videos). Each video contains about 300-600 frames, comprising of large pose and expression variations and significant head rotations. The second dataset, CMU MoBo is a commonly used benchmark dataset for video-based face recognition consisting of 96 sequences of 24 different subjects (each person has 4 videos). Each video contains about 300 frames. For both datasets, faces were extracted using the Viola-Jones cascaded face detector [16, resized to grayscale images of $32 \times 32$ pixels, followed by histogram equalization to remove illumination effects. Some sample images are shown in Fig. 2 ,

For each subject, one video sequence is used for training, and the remaining video sequences for testing. To ensure extensive evaluation on the datasets, we construct our test set by randomly sampling 20 sub-sequences consisting of 100 frames from each test video sequence. The test sequences are evaluated using a probabilistic Bayes classifier where the class with the maximum posterior probability, $c^{*}=\arg \max P\left(c \mid x_{1}, \ldots, x_{N}\right)$ is the identified subject.

We use 7 exemplars for Honda/UCSD and 6 exemplars for MoBd1, while intra-class and inter-class neighbors are fixed at $k=k^{\prime}=M-1$. The tuning parameter is set at $\beta=0.75$ for all our experiments. It should be noted that the optimal number of feature dimensions for all methods were determined empirically through experiments.

\footnotetext{
$\overline{1}$ The number of exemplars selected from each video is heuristically determined from the residual error curve of clustering distance criterion [13.
} 


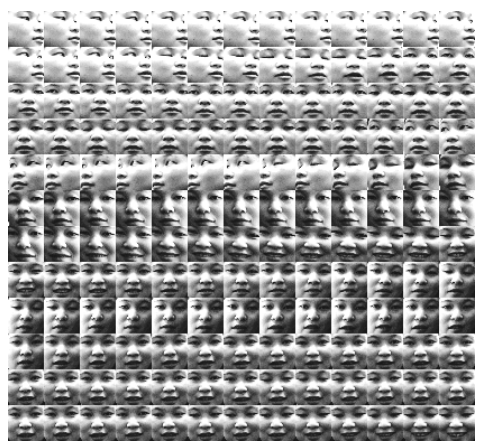

(a) Honda/UCSD

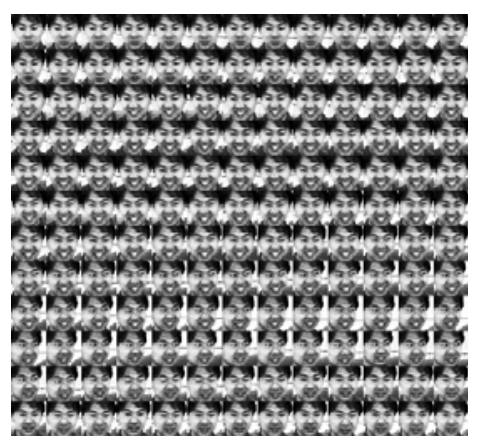

(b) CMU MoBo

Fig. 2. Sample images extracted from a video sequence of different datasets

\subsection{Comparative Evaluation}

The proposed NDMP method is compared against other classical (PCA, LDA) and recent state-of-art methods (LPP, MFA, NPE). The overall recognition performance on both Honda/UCSD and CMU MoBo datasets is summarized in Table1, which shows that the NDMP method can outperform other methods in recognizing faces in video sequences. The strength and robustness of NDMP over the rest is more apparent in the Honda/UCSD data set, where video sequences possess a wide range of complex face poses and head rotations. Interestingly, LPP and MFA performed rather poorly in this dataset. The constraint tuning parameter, $\beta$ in the generalized NDMP (see Eq. (19)) can be tuned to values between 0 and 1 to adjust the contribution of the local neighborhood constraint and global invariance constraint. From Fig. 3, we can observe that while both constraints seemed equally important, slightly better results can be expected by imposing more influence towards constraining the local neighborhood structure.

\subsection{Rank-Based Identification Setting}

To further evaluate the reliability of the NDMP method in a rank-based identification setting, we present its performance using a cumulative match curve (CMC). To accommodate this evaluation setting, we adopt a probabilistic voting strategy where the top $n$ matches based on posterior probability scores are given a vote at each frame. The class with the majority vote is identified as the subject in the test sequence.

The CMCs of various methods evaluated on both datasets in Fig. 4 showed that NDMP consistently yielded better recognition rates throughout rank- $n$ top matches for the Honda/UCSD. It also achieved a perfect recognition score (100\%) for the CMU MoBo dataset with the top 3 matches. In contrast, global 
Table 1. Recognition rates (\%) of various manifold learning methods on the evaluated datasets

\begin{tabular}{lcccccc}
\hline \multicolumn{1}{c}{ Datasets } & \multirow{5}{c}{ Methods } \\
& PCA & LDA & LPP & MFA & NPE & NDMP \\
\hline \hline Honda/UCSD & 60.7 & 68.9 & 56.8 & 57.4 & 71.7 & 86.9 \\
CMU MoBo & 86.6 & 92.6 & 89.3 & 91.4 & 96.3 & 97.7 \\
\hline
\end{tabular}

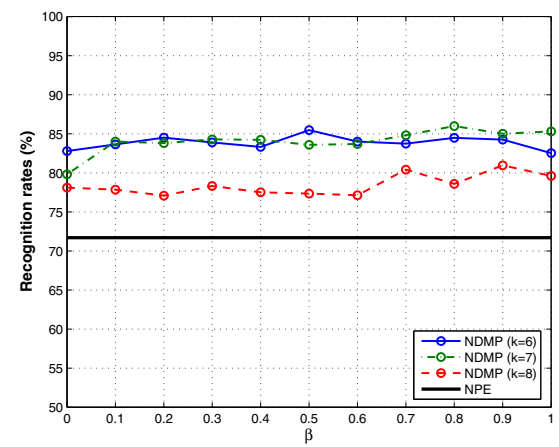

Fig. 3. Comparison of different values of $\beta$ on the Honda/UCSD dataset

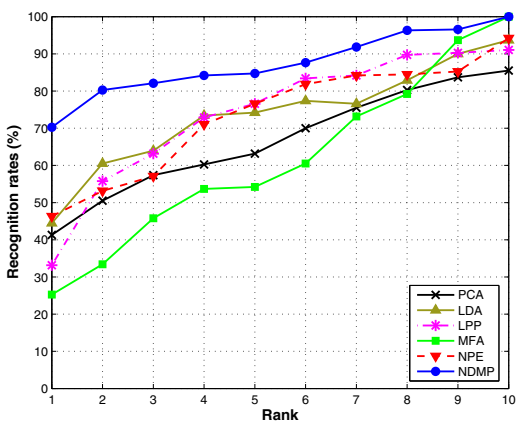

(a) Honda/UCSD

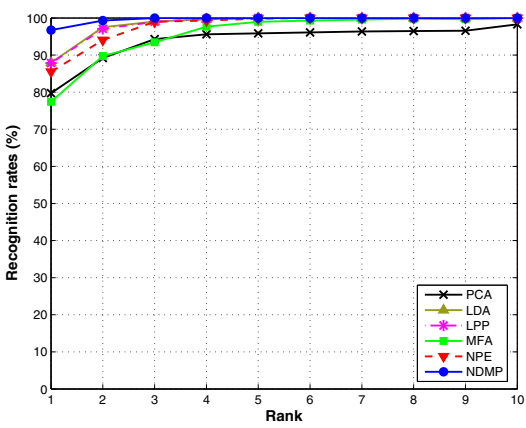

(b) CMU MoBo

Fig. 4. Comparison of cumulative match curves of various methods

methods such as PCA and LDA performed poorly due to their inability to learn the nonlinear manifold of appearance variations that is inherent in videos. It can be observed that the performance of other local neighborhood-preserving methods (MFA, LPP, NPE) tend to improve rapidly as the rank increases. 


\section{Conclusion}

In this paper, we present a novel supervised manifold learning method called Neighborhood Discriminative Manifold Projection (NDMP) for video-based face recognition. The NDMP method constructs a discriminative eigenspace projection from nonlinear face manifolds based on both local neighborhood geometry and global manifold structure. Extensive experiments on standard video face datasets demonstrated the robustness and effectiveness of the proposed method compared to classical and recent state-of-art methods. In the future, the NDMP method can be further generalized to a nonlinear form in kernel feature space. Its potential usage in practical real-world applications is also worth exploring.

Acknowledgments. The authors wish to thank all who had provided invaluable suggestions throughout the development of this work. The authors also pay tribute to Sam Roweis, co-author of LLE, on his passing away in early 2010.

\section{References}

1. Brand, M.: Charting a manifold. In: Proc. of NIPS 15, pp. 961-968 (2003)

2. Turk, M., Pentland, A.: Eigenfaces for recognition. J. Cogn. Neurosc. 3(1), 71-86 (1991)

3. Cox, T.F., Cox, M.A.A.: Multidimensional Scaling. Chapman and Hall, Boca Raton (2001)

4. Belhumeur, P.N., Hespanha, J.P., Kriegman, D.: Eigenfaces vs Fisherfaces: Recognition using class specific linear projection. IEEE Trans. PAMI 19, 711-720 (1997)

5. Roweis, S.T., Saul, L.: Nonlinear dimensionality reduction by locally linear embedding. Science 290, 2323-2326 (2000)

6. Tenenbaum, J.B., de Silva, V., Langford, J.C.: A global geometric framework for nonlinear dimensionality reduction. Science 290, 2319-2323 (2000)

7. Hadid, A., Peitikäinen, M.: From still iamge to video-based face recognition: An experimental analysis. In: IEEE FG, pp. 813-818 (2004)

8. Fan, W., Wang, Y., Tan, T.: Video-based face recognition using bayesian inference model. In: Kanade, T., Jain, A., Ratha, N.K. (eds.) AVBPA 2005. LNCS, vol. 3546, pp. 122-130. Springer, Heidelberg (2005)

9. He, X.F., Niyogi, P.: Locality preserving projections. In: Proc. of NIPS 16, pp. 153-160 (2003)

10. Yan, S., Xu, D., Zhang, B., Zhang, H.J., Yang, Q., Lin, S.: Graph embedding: A general framework for dimensionality reduction. IEEE Transactions on PAMI 29(1), 40-51 (2007)

11. He, X., Cai, D., Yan, S., Zhang, H.J.: Neighborhood preserving embedding. In: IEEE ICCV, pp. 1208-1213 (2005)

12. Gross, R.: Face databases. In: Li, S.Z., Jain, A.K. (eds.) Handbook of Face Recognition, Springer, Heidelberg (2005)

13. Duda, R., Hart, P., Stork, D.: Pattern Classification. Wiley, Chichester (2000)

14. Lee, K.C., Ho, J., Yang, M.H., Kriegman, D.: Visual tracking and recognition using probabilistic appearance manifolds. CVIU 99(3), 303-331 (2005)

15. Gross, R., Shi, J.: The CMU Motion of Body (MoBo) Database. Technical Report CMU-RI-TR-01-18, Robotics Institute, CMU (2001)

16. Viola, P.: Jones. M.: Rapid object detection using a boosted cascade of simple features. In: CVPR, pp. 511-518 (2001) 